\title{
A bulk silicon micromachined structure for gas microdischarge-based detection of beta-particles
}

\author{
Christine K Eun, Chester Wilson ${ }^{1}$ and Yogesh B Gianchandani \\ Department of Electrical Engineering and Computer Science, University of Michigan, Ann Arbor, \\ MI 48109, USA \\ E-mail: eunc@umich.edu
}

Received 21 May 2008, in final form 29 June 2008

Published 24 July 2008

Online at stacks.iop.org/JMM/18/095007

\begin{abstract}
This paper describes two Si micromachined structures for sensing beta-particles. The basic device (the micro-detector) includes a square silicon cathode surrounded by a concentric anode, and is formed by stacks of glass and Si wafers. Incident beta-particles ionize the gas encapsulated between the electrodes, resulting in an avalanche current pulse or 'count'. It is shown experimentally that devices with $8 \times 8 \mathrm{~mm}^{2}$ footprint can detect radiation in the proximity of sealed sources, such as ${ }^{90} \mathrm{Sr}$ and ${ }^{204} \mathrm{Tl}$ with $0.1-1.0 \mu \mathrm{Ci}$ strengths. The sensitivity $\left(\mathrm{cpm} \mathrm{mRad}{ }^{-1} \mathrm{~h}\right)$ of the micromachined device is comparable to that of commercial radiation detectors, but substantially superior when normalized to the detector volume, which is about $0.06 \%$ of the conventional detectors. An extension of the basic device, the stacked micro-detector, consists of a two-tiered arrangement of cavities separated by a thin glass intercavity attenuator that is intended to provide controlled energy absorption. Higher energy particles are detected in both cavities, while lower energy particles are detected in the first cavity and subsequently absorbed by the intercavity attenuator. This can provide initial assessment of the incident radiation without adding significant complexity to the system. Preliminary experimental validation is performed by comparing the device response to ${ }^{204} \mathrm{Tl}$ and ${ }^{90} \mathrm{Sr}$.
\end{abstract}

(Some figures in this article are in colour only in the electronic version)

\section{Introduction}

Environmental monitoring is emerging as a significant driver of microsystems technology. One sensor category of particular interest is radiation sensing. There is a rising demand for microsystems that can provide real-time, first-alert information on the presence of dangerous radioisotopes. In particular, these miniaturized detectors can target applications ranging from monitoring radiation safety levels of nuclear power plants to guarding against illicit trafficking of radioactive chemicals and port screening for homeland security.

The miniaturization of radiation detectors can lead to ultra-portable and reconfigurable sensor systems, lower

${ }^{1} \mathrm{C}$ Wilson was formerly with the University of Michigan. He is presently with the Institute for Micromanufacturing, Louisiana Tech University, USA. power requirements, and permit the use of lithographic manufacturing to drive down sensor cost. In addition, smaller sensor dimensions allow for the formation of composite sensor structures, which can increase overall functionality. Finally, scaling down feature sizes and electrode spacing can lead to increased resolution in imaging applications.

Beta-particles are essentially high-energy electrons that can travel distances on the order of meters in air. A wide variety of source isotopes release beta-particles during radioactive decay. Each isotope has a unique and continuous energy spectrum with a characteristic maximum 'endpoint energy' or maximum possible energy the radioisotope can emit. For example, ${ }^{204} \mathrm{Tl}$ produces a spectrum of beta-particles with an endpoint energy of $0.764 \mathrm{MeV}$. Another pure beta-emitter, ${ }^{90} \mathrm{Sr}$, is a particularly hazardous material, which can emit up to $0.546 \mathrm{MeV}$ beta-particles. Its toxicity arises less from 
the radiation energy and more from its chemical nature. It is easily absorbed into the human body, where it displaces calcium in bone and remains there with a radioactive halflife of 27 years. ${ }^{90} \mathrm{Sr}$ decays into ${ }^{90} \mathrm{Y}$, which is also a betaemitter, and has an endpoint energy of $2.280 \mathrm{MeV}$ [1]. Its half-life is $64 \mathrm{~h}$, which is significantly shorter than that of ${ }^{90} \mathrm{Sr}$. Each beta-particle emanating from a ${ }^{90} \mathrm{Sr}$ sample is also accompanied by another beta-particle emanating from the ${ }^{90} \mathrm{Y}$ that is consequently produced.

Other products of radioactive decay include alphaparticles and gamma-rays. Alpha-particles have a relatively large mass, which prevents them from reaching high velocities. Gamma-rays are extremely small, high-energy photons characterized as electromagnetic radiation with the highest frequency and energy. Each presents unique detection challenges. Alpha-particles are easily masked, while gammarays have a greater range but very low interaction probability. Oftentimes, when a radioisotope undergoes decay, betaparticles are produced in conjunction with alpha-particles and gamma-rays. ${ }^{60} \mathrm{Co}$ (used in radiotherapy) emits beta-particles with an endpoint energy of $0.318 \mathrm{MeV}$ and gamma-rays at specific, discrete energy levels (1.17 MeV and $1.33 \mathrm{MeV})$. We have chosen to concentrate on beta activity for our efforts because most of the radioactive materials of interest in medical and other commercial applications involve beta decay.

There are primarily three types of radiation detectors that are commercially available at present: scintillators, solid-state and gas-based detectors [2]. In recent years, each method has seen improvements enabled by microfabrication technologies. Some micromachined scintillators use scintillating crystals deposited directly into thin-film silicon-well photodetectors for gamma and neutron imaging [3, 4]. Other scintillation technologies have utilized new materials with enhanced performance characteristics (e.g., high light output and fast response time) [5, 6]. The main advantages of scintillator technology include the ability to perform energy spectroscopy and flexibility in producing various crystal sizes and shapes. Potential disadvantages include limited energy resolution, the need for photo-multiplier (PM) tubes, power requirements and temperature dependence [2].

Solid-state detectors directly convert ionizing radiation into electrical pulses, achieving energy resolution superior to scintillators [7-9]. However, some require cryogenic cooling to distinguish radiation type and energy and can be susceptible to performance degradation due to radiation damage [10]. One type of solid-state device uses pixelated silicon structures at room temperature to provide spatial imaging of beta-particle flux [11]. The latest solid-state detector technologies use wide band gap, high-Z materials such as $\mathrm{CdZnTe}$, which offers high conversion efficiency, and operates at room temperature $[12,13]$.

Gas-based detectors (e.g., Geiger counters) are often favored for environmental surveillance efforts (e.g., in looking for radiation leaks and inadvertent contamination) [2]. They are relatively simple and robust, operate over a large temperature range, and measure a wide range of radiation species and energies. Typically, these detectors involve biased electrodes (anode and cathode) enclosed within a gas-filled chamber. Ionizing radiation (e.g., beta-particles) interacts with the gas, and the resulting electron avalanches create a current pulse that registers as a 'count'.

Gas-based sensing of gamma radiation is facilitated by using high-pressure, large atomic number fill-gases [14] and by encapsulating the gas and electrodes within metal walls that convert incident photons into photo-electrons through the photoelectric effect and Compton scattering.

One inherent limitation of conventional Geiger counters is that they cannot distinguish between different radioactive species (e.g., between benign and lethal isotopes). This function is performed by proportional counters, which are similar to Geiger counters, but biased at a lower voltage such that the magnitude of the current pulses is proportional to the energy of the ionizing radiation. Count rates and pulse heights are collected, and over time the unique energy profile of the radioisotope is mapped out. The electronic interface for proportional counters is, therefore, significantly more complex than for Geiger counters.

One of the first reported micropatterned gaseous detectors used photolithographically manufactured precise, planar electrode structures in microstrip gas chambers (MSGC) $[15,16]$. Other micro-fabricated, gas-based detectors included the MICROMEGAS [17], the micro-CAT [18] and the gas electron multiplier (GEM) [19]. Focusing on high spatial resolution, each had been mainly targeted for position sensing applications, such as medical imaging and nuclear particle tracking, and not necessarily intended for environmental monitoring.

In this paper, we first describe (in section 2) a lithographically microfabricated $\mathrm{Si}$ /glass micro-detector that uses a bulk micromachined glass-silicon-glass sandwich to form detection cavities and sensing electrodes ${ }^{2}$. An extension of the basic device (described in section 3) uses a twotiered arrangement of stacked micro-detectors so that incident beta-particles pass through two successive detection cavities separated by a glass intercavity attenuator intended to provide controlled energy absorption ${ }^{3}$. A differential measurement can potentially provide a preliminary indication of the radioactive species without the need for additional processing electronics, which results in greater portability and power savings. Also addressed is the energy attenuation of beta-particles through various thicknesses of the intercavity attenuator. Section 4 details the fabrication process for the micro-detector and the stacked micro-detector, while section 5 presents experimental results.

\section{Basic device and concepts}

\subsection{Device description}

The basic micro-detector structure consists of a square chamber with a central cathode and a peripheral anode (figure 1(a)). The region proximal to the cathode has a

2 Portions of this work have been reported in conference abstract form in [20].

3 Portions of this work have been reported in conference abstract form in [21]. 


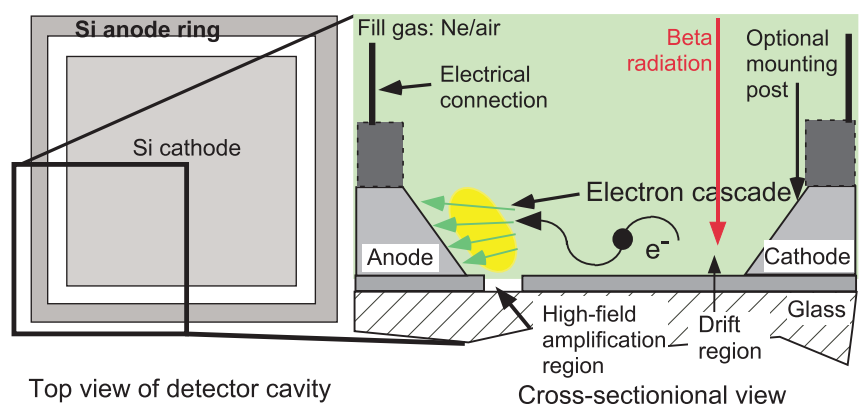

(a)

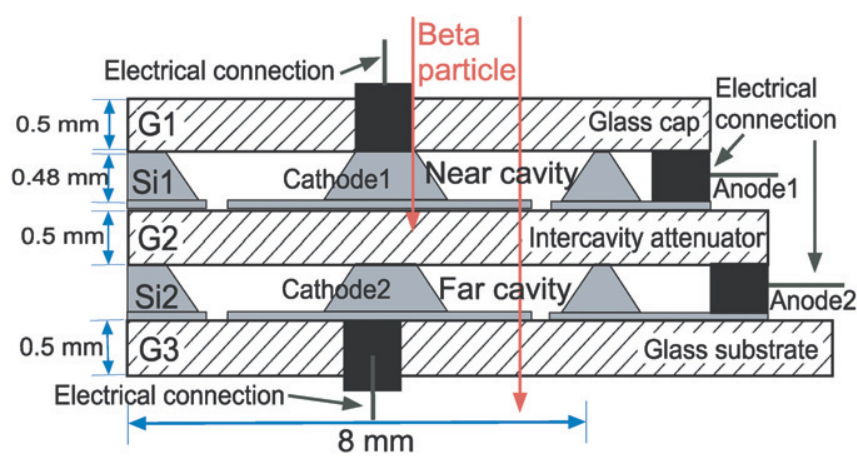

(b)

Figure 1. (a) The basic micro-detector structure utilizes dissolved silicon bonded to glass as the anode-cathode configuration. Beta radiation passing through the drift region creates liberated electrons, which are accelerated in the amplification region and create an electron cascade. (b) The stacked micro-detector uses a two-tiered structure with aligned cavities, as well as, two separate electrical connections to the cathodes. There is a potential for estimating the beta-particle energy by taking a differential measurement of the count rates in the two cavities.

weak electric field and is called the drift region, whereas that adjacent to the anode is the high-field amplification region. Beta-particles enter the detection cavity and ionize the fill-gas in the drift region. (In contrast with most conventional Geiger counters, this gas is at atmospheric pressure.) The electrons are slowly accelerated into the amplification region. In the amplification region, the electrons are quickly accelerated through a higher field region resulting in an electron cascade. Designing the drift region to be much larger than the amplification region allows pulses that are independent of the entry position of the beta-particle.

The device consists of an $8 \times 8 \mathrm{~mm}^{2}$ cavity formed in Si. The cavity depth is approximately $473 \mu \mathrm{m}$. The silicon electrodes are bonded to a $500 \mu \mathrm{m}$ thick Pyrex glass substrate. The inner electrode (cathode) is a $22 \mu \mathrm{m}$ thick boron-doped $\mathrm{p}^{++} \mathrm{Si}$, while the $473 \mu \mathrm{m}$ thick surrounding wall structure (anode) is primarily bulk Si. However, underneath the wall structure is a $22 \mu \mathrm{m}$ thick layer of $\mathrm{p}^{++} \mathrm{Si}$ that defines the amplification region (approximately $500 \mu \mathrm{m}$ long). The drift region is approximately $4 \mathrm{~mm}$ long and is located between the center of the cavity to the anode wall. A glass cap covers the device.

According to Paschen's curve, which describes the nonlinear relationship between gaseous breakdown potential and the product of fill-gas pressure and gap spacing, scaling down the feature sizes (i.e., the gap spacing) allows the
Table 1. Electric field modeling for the micro-detector was performed with ANSYS ${ }^{\circledR}$ 10.0. The maximum electric field strength was $3.8 \times 10^{8} \mathrm{~V} \mathrm{~m}^{-1}$ located at the corners of the electrodes.

\begin{tabular}{ll}
\hline Device specifications & \\
\hline Cathode length & $700 \mu \mathrm{m}$ \\
Gap spacing & $500 \mu \mathrm{m}$ \\
Width of sloped side wall & $340 \mu \mathrm{m}$ \\
Max. height of anode wall & $500 \mu \mathrm{m}$ \\
Height of cathode layer & $100 \mu \mathrm{m}$ \\
Angle of sloped wall & $\sim 54.7^{\circ}$ \\
\hline
\end{tabular}

pressure in the detection cavity to be set close to atmospheric pressure. This offers two advantages. First, the increased density of fill-gas atoms increases the probability of interaction with beta-particles. Second, the reduced pressure differential to the ambient lowers the mechanical load on the cavity walls. In principle, this can justify the use of thinner walls, thereby reducing beta energy attenuation and enabling the detection of lower energy beta-particles.

For common fill-gases (e.g., $\mathrm{Ne}$ and $\mathrm{He}$ ), at atmospheric pressure, the threshold electric field for gas multiplication is on the order of $10^{6} \mathrm{~V} \mathrm{~m}^{-1}$ [2]. Typical Geiger counters operate with electric fields, near the high field anode wire, on the order of around a few $10^{6} \mathrm{~V} \mathrm{~m}^{-1}$. Applying a potential $\left(V_{\text {applied }}\right)$ of $800 \mathrm{~V}$ between a gap spacing of $500 \mu \mathrm{m}$, the electric field is approximately $1.6 \times 10^{6} \mathrm{~V} \mathrm{~m}^{-1}$. A threedimensional simulation using ANSYS 10.0 shows that the maximum electric field $\left(3.8 \times 10^{8} \mathrm{~V} \mathrm{~m}^{-1}\right)$ is located in the amplification region (near the corners of the cathode) and exceeds the minimum operating field $\left(\sim 10^{6} \mathrm{~V} \mathrm{~m}^{-1}\right)$. The electric field in the drift region (gap spacing of $4 \mathrm{~mm}$ ) is approximately $2 \times 10^{5} \mathrm{~V} \mathrm{~m}^{-1}$. The device specifications for the model are given in table 1 .

\subsection{Regions of operation}

Based on the bias voltage and the resulting discharge characteristics, detectors fall into four regimes of operation: the ion saturation regime, the proportional regime, the limited proportional regime and the Geiger-Mueller regime. (These regimes apply to gas-based sensors of both beta-particles and photons like x-rays and gamma radiation.) The regime with the lowest voltage is the ion saturation region, in which the only charge collected is from gas directly ionized by impinging radiation.

At a higher bias between the anode and cathode, gas multiplication begins to occur and the amount of collected current increases; this is the proportional region. The amount of current is roughly proportional to the energy of the impinging radiation. Proportional counters are typically used to perform spectroscopy on soft $\mathrm{x}$-rays and gammarays by measuring the photo-electrons emitted from photon interactions within the detector wall. In contrast, impinging beta-particles mostly interact with the fill-gas and impart a portion of their kinetic energy to the ionization process. At yet higher bias values is the limited proportional region. The electric field becomes distorted and causes nonlinear effects 
on the current pulses. The current pulse amplitude becomes less dependent on the energy of the radiation.

At even higher bias values, the device operates in the Geiger-Mueller region. Every beta-particle triggers an avalanche process that ionizes the entire gas volume and a complete gaseous discharge is generated. While electrons are quickly collected at the anode, the slower moving ions create a space-charge region that surrounds the cathode. (Similar space-charge regions have been reported in DC microplasmas [22].) The applied field decreases below the threshold for multiplication and the discharge is quenched. Consequently, the current pulses are approximately uniform in size and independent of the radiation energy. For conventionally-sized detectors, the pulses are also large enough in amplitude that they can be detected without further signal amplification.

Past implementations of micro-scale radiation detectors, with gap spacings on the order of tens to hundreds of microns, have produced current pulses that range in amplitude from $0.1 \mathrm{nA}$ to $10 \mu \mathrm{A}$ (pre-amplification) and typically require further amplification and signal processing [15, 19, 23, 24]. This micro-detector design operates near the Geiger-Mueller regime with current pulses on the order of 1-100 mA, which are easily detected. The avalanche multiplication of charge that takes place in the gas breakdown is thought to be further assisted by secondary emission from the anode. This may compensate for the relatively small size of the amplification region compared to traditional devices.

\subsection{Characterizing detector performance}

The standard generally used to characterize radiation detectors includes parameters such as sensitivity, operating voltage and detector volume. Sensitivity is the count rate (per minute) obtained by the detector divided by the dose rate $\left(\mathrm{mRad} \mathrm{h}^{-1}\right)$ for beta radiation from the calibration source [25]. Dose rates are dependent on beta-particle fluence or flux, average beta energy, and the beta absorption coefficient. The fluence rate is given by [25]

$$
\Phi=\frac{A f}{4 \pi r^{2}}\left(\text { disintegrations } \mathrm{m}^{-2} \mathrm{~s}^{-1}\right)
$$

where $A$ is the source activity (in units of $\mathrm{Bq}$, or disintegrations per second), $f$ is the fractional yield of radiation emitted with the average energy, $\bar{E}(\mathrm{MeV})$, and $r$ is the distance from the source to the detector $(\mathrm{cm})$. The beta absorption coefficient for the fill-gases relevant to this work (i.e., $\mathrm{Ne}, \mathrm{He}$ and $\mathrm{He}$ /air) has been approximated by the absorption coefficient of air [25]:

$$
\mu_{\beta, \text { air }}=16\left(E_{\beta, \max }-0.036\right)^{-1.4}\left(\mathrm{~cm}^{2} \mathrm{~g}^{-1}\right)
$$

where $E_{\beta \text {, max }}$ is the endpoint energy.

The beta radiation dose rate, $\dot{D}_{\beta}$, for beta-particles is given by [25]

$$
\begin{aligned}
& \dot{D}_{\beta}= \\
& \frac{1000 A f \bar{E}\left(1.602 \times 10^{-6} \mathrm{erg} \mathrm{MeV}^{-1}\right) \mu_{\beta}\left(\mathrm{cm}^{2} \mathrm{~g}^{-1}\right)\left(3600 \mathrm{sh}^{-1}\right)}{\left(4 \pi r^{2}\right) 100\left(\mathrm{erg} \mathrm{g}^{-1} \mathrm{rad}^{-1}\right)}\left(\mathrm{mad} \mathrm{h}^{-1}\right) \\
& \quad \dot{D}_{\beta}=5.768 \times 10^{-5} \frac{1000 A f \bar{E} \mu_{\beta}}{4 \pi r^{2}}\left(\mathrm{mRad} \mathrm{h}^{-1}\right) .
\end{aligned}
$$

For the purpose of this paper, sensitivity is given by

$$
\text { sensitivity }=\frac{\text { count rate }(\mathrm{cpm})}{\dot{D}_{\beta}\left(\mathrm{mRad} \mathrm{h}^{-1}\right)}\left(\mathrm{cpm} \mathrm{mRad}{ }^{-1} \mathrm{~h}\right)
$$

where count rate is experimentally determined.

\section{The stacked micro-detector}

As an extension of the basic device, the stacked microdetector uses a two-tiered structure with aligned cavities (figure 1(b)). The glass intercavity attenuator between the two cavities (near and far) is intended to provide controlled energy absorption. Lower energy beta-particles are detected in the near cavity alone, and then stopped by the intercavity attenuator. The higher energy particles are expected to have enough energy to pass through the attenuator and be detected in both cavities. The attenuator can have varying thicknesses, allowing customization to a particular energy range.

There are two main mechanisms of energy loss as a betaparticle passes through a material, collisional and radiative. For lower energy beta-particles, collisional energy loss is dominant. Also known as the 'stopping power' of a material, the energy loss is given by [26]

$$
\begin{gathered}
-\frac{\mathrm{d} E}{\mathrm{~d} x} \text { collisional } \\
=\frac{4 \pi k_{0}^{2} e^{4} n}{m_{o} c^{2} \beta^{2}}\left[\ln \frac{m_{o} c^{2} \tau \sqrt{\tau+2}}{\sqrt{2} I}+F(\beta)\right]\left(\mathrm{keV} \mathrm{cm}^{-1}\right) \\
F(\beta)=\frac{1-\beta^{2}}{2}\left[1+\frac{\tau^{2}}{8}-(2 \tau+1) \ln 2\right] \\
\tau=\frac{T}{m c^{2}},
\end{gathered}
$$

where $I$ is the mean excitation energy of the medium $(\mathrm{eV}), \beta$ is the beta-particle velocity given as a fraction of the speed of light, $m_{o}$ is the mass of an electron $(\mathrm{kg}), c$ is the speed of light $\left(\mathrm{m} \mathrm{s}^{-1}\right), T$ is the kinetic energy of the beta-particle and $n$ is the number of electrons per unit volume in the medium $\left(\mathrm{m}^{-3}\right)$. Since the parameter $n$ is difficult to calculate for materials with complex compositions such as Pyrex glass, this value was estimated by fitting the theoretical estimate with reference data given in [27]. The beta energy spectra for ${ }^{90} \mathrm{Sr} /{ }^{90} \mathrm{Y}$ and ${ }^{204} \mathrm{Tl}$ are shown in figure 2(a) [28]. It is based on theoretical data and is accurate except at very low energies. Each spectrum illustrates the statistical distribution of beta energy from a total of $10^{6}$ decays [29]. Figure $2(b)$ shows the stopping power of glass as a function of beta energy. Table 2 summarizes the parameter values used in the analytical estimate. The agreement between the estimate and the LDNL experimental data is within $1.5 \%$.

A simple beta model provides a rough guideline for the expected count ratio in the stacked micro-detector. Count ratio is defined as the fraction of counts in the far cavity (bottom cavity) divided by the fraction of counts in the near cavity (top cavity). Using the beta energy spectrum and the energy attenuation through the glass intercavity attenuator, the count ratio can be estimated. The assumptions and 


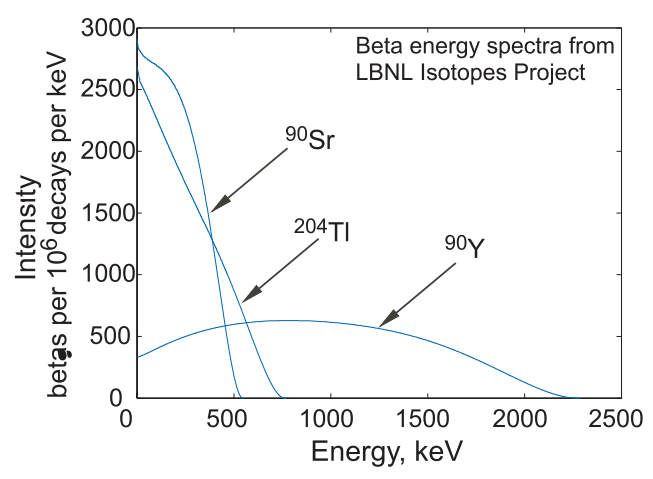

(a)

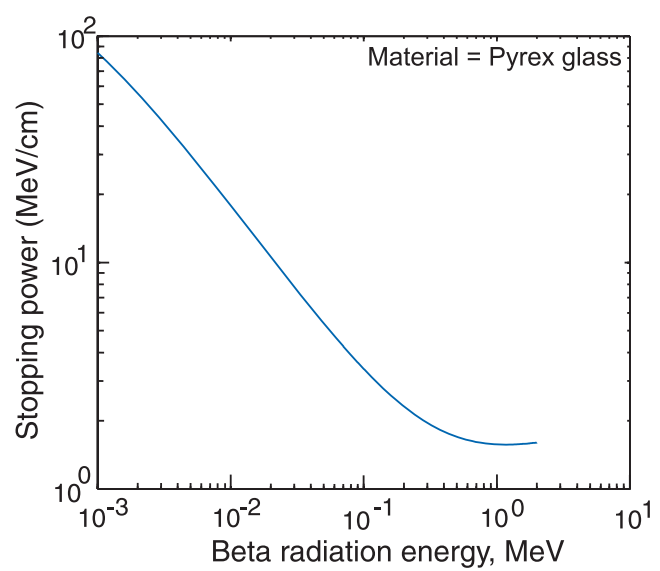

(b)

Figure 2. (a) Beta energy spectra for various chemical isotopes showing unique profiles and endpoint energies [28, 29]. (b) Theoretical estimate of stopping power as a function of beta energy through glass. The difference between the theoretical estimate and published experimental data [27] was found to be within $1.5 \%$. Stopping power is defined as the thickness of a material needed to completely absorb the beta energy.

Table 2. Parameters used for calculating beta energy loss through glass.

\begin{tabular}{llll}
\hline Parameter & Symbol & Value & Units \\
\hline Mean excitation energy & $I$ & 134 & $\mathrm{eV}$ \\
Mass of electron & $m_{0}$ & $9.11 \times 10^{-31}$ & $\mathrm{~kg}$ \\
Speed of light & $c$ & $3 \times 10^{10}$ & $\mathrm{~cm} \mathrm{~s}^{-1}$ \\
Electron charge & $e$ & $1.602 \times 10^{-19}$ & $\mathrm{C}$ \\
$\begin{array}{l}\text { Number of electrons per } \\
\text { unit volume in the medium }\end{array}$ & $n$ & $3 \times 10^{29}$ & $\mathrm{~m}^{-3}$ \\
\hline
\end{tabular}

simplifications are discussed in detail in section 6. There are two device-specific fitting parameters: $E_{C 1}$ and $E_{C 2} . E_{C 1}$ is the equivalent minimum energy a beta-particle requires to instigate an avalanche breakdown that results in a 'count'. Several values of $E_{C 1}$ are plotted as a function of glass intercavity attenuator thickness for various isotopes (figure 3 ). The parameter $E_{C 2}$ represents the equivalent upper limit of the beta energy, above which the particle is unlikely to transfer enough energy to the fill-gas to initiate a breakdown.

$E_{C 2}$ is related to the number of ions produced per unit length of travel by the beta-particle, also known as the primary ionization (PI). At high beta energies, the PI decreases and beta-particles tend to transfer (on average) a lower amount of energy as they travel through a medium [30, 31]. For beta velocities (in units of the velocity of light) ranging from $\beta=$ 0.45 to $\beta=0.96$, the PI varies with $1 / \beta^{2}$ [32]. For $\beta \approx$ 0.76 , which corresponds to a beta energy of $270 \mathrm{keV}$, the PI is approximately 26.1 ions $\mathrm{cm}^{-1}$ for $\mathrm{N}_{2}$ and 18.3 ions $\mathrm{cm}^{-1}$ for $\mathrm{Ne}$ at normal temperature and pressure [30]. For the travel length of $500 \mu \mathrm{m}$ (e.g., the height of the micro-detector), a betaparticle with a velocity of 0.76 will approximately generate on average 1.3 ions in $\mathrm{N}_{2}$ and 0.92 ions in $\mathrm{Ne}$.

In the beta model, the theoretical estimate of the count rate in the near cavity is calculated by: (1) summing the number of beta-particles with energies from $E_{C 1}$ to $E_{C 2}$ and (2) subtracting the number of particles that are attenuated through

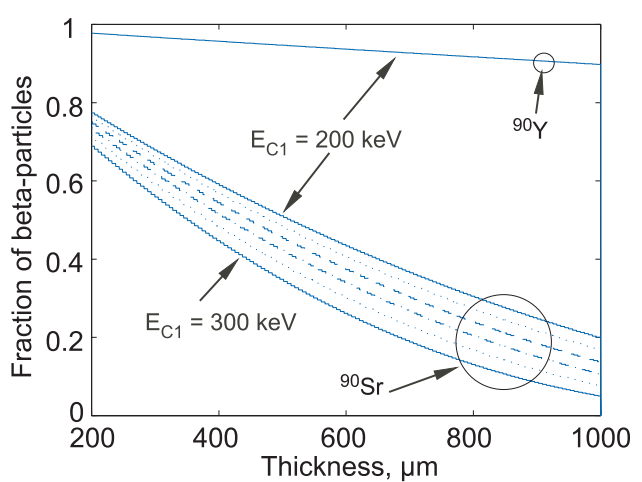

(a)

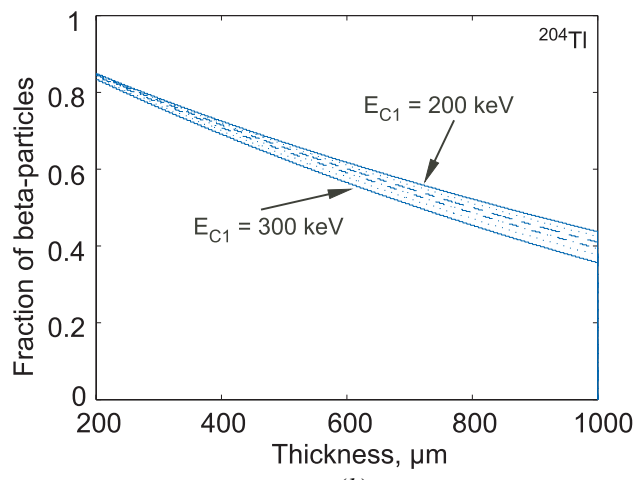

(b)

Figure 3. Theoretical estimates of the fraction of beta-particles that pass through the glass versus those absorbed in the glass as a function of glass thickness for $(a){ }^{90} \mathrm{Sr}$ and its daughter isotope ${ }^{90} \mathrm{Y}$ and $(b)^{204}$ Tl. For each trend, $E_{C 1}$ has been plotted from 200$300 \mathrm{keV}$, in $20 \mathrm{keV}$ intervals. Varying trends for each isotope are attributed to differences in the beta spectrum shape and endpoint energies, which can potentially be used to differentiate between radioactive isotopes.

the top glass cap. The count rate in the far cavity is calculated similarly, but also takes into account the additional energy loss through the intercavity attenuator. By matching experimental 


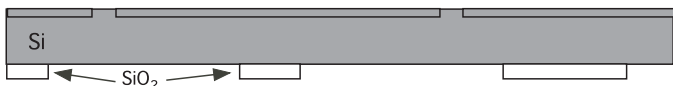

1. Si wafer boron implanted, oxide patterned

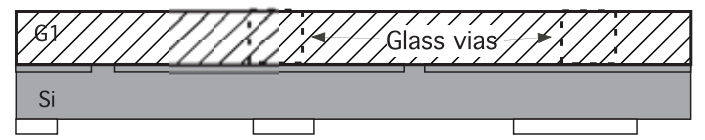

2. Etch glass vias, Si wafer anodically bonded to glass

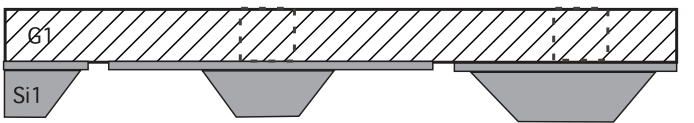

3. Si wafer EDP etched, oxide removed

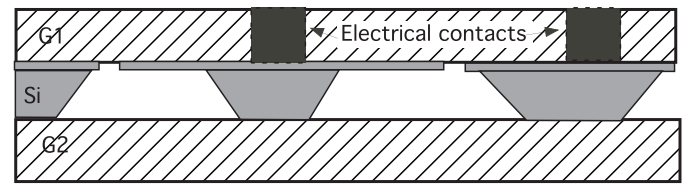

4. Wafer stacked on glass cap

Figure 4. Process flow of the micro-detector. Mask 1 defines a boron etch stop and mask 2 a patterned oxide. Machined glass vias allow electrical contact to the cathode and anode. Anodic bonding followed by EDP etching defines the device cavity. The processed $\mathrm{Si}$ wafer stacked on the glass cap completes the micro-detector structure.

count ratios with theoretical estimates for a known attenuator thickness, an initial real-time assessment of the isotope identity can be provided.

\section{Device fabrication}

The micro-detector is fabricated in a simple, two-mask process involving a $\mathrm{Si}$ wafer and two glass wafers-G1 and G2 (figure 4). Beginning with a $500 \mu \mathrm{m}$ thick $\langle 100\rangle \mathrm{Si}$ wafer, a $1 \mu \mathrm{m}$ thick $\mathrm{SiO}_{2}$ is grown using wet oxidation. Mask 1 defines a $\mathrm{p}^{++} \mathrm{Si}$ boron diffusion profile for the central cathode and amplification region near the anode wall. Boron is diffused $8 \mu \mathrm{m}$ deep and the oxide is removed with a hydrofluoric acid (HF) dip. Another $1 \mu \mathrm{m}$ thick layer of $\mathrm{SiO}_{2}$ is grown on the $\mathrm{Si}$ wafer and patterned by Mask 2, which defines the footprint for the tapered anode wall. The boron doping of silicon is used as an etch-stop during the dissolution of undoped $\mathrm{Si}$ in ethylene diamine pyrocatechol (EDP). EDP is a wet solvent that anisotropically etches silicon, exhibits dopant selectivity and is widely used in the microfabrication of sensors [33].

A $500 \mu \mathrm{m}$ thick Pyrex (Corning 7740) glass wafer (G1) is perforated in locations that will later provide electrical access to the cathode. While we prefer electrochemical discharge machining for perforating the glass, other options are available [34-36].

The Si wafer is aligned to G1 and anodically bonded to it. The Si wafer is etched using EDP, forming the cavity structures and exposing the boron-doped cathodes and amplification regions. Another $500 \mu \mathrm{m}$ thick Pyrex glass wafer (G2) is machined to form the glass cap, which is stacked on the cavities of the Si/G1 structure. The entire structure is then tested in a flow chamber with various fill-gases. A metal layer (e.g., Au) can be optionally patterned on the glass cap to enable

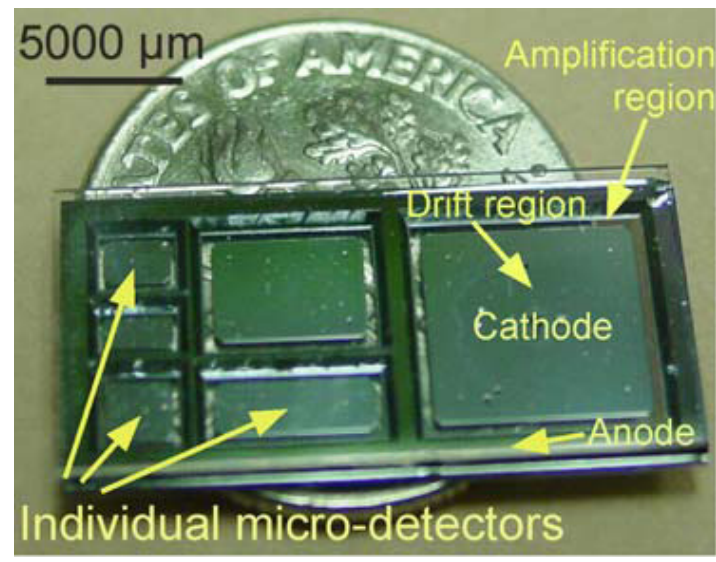

(a)

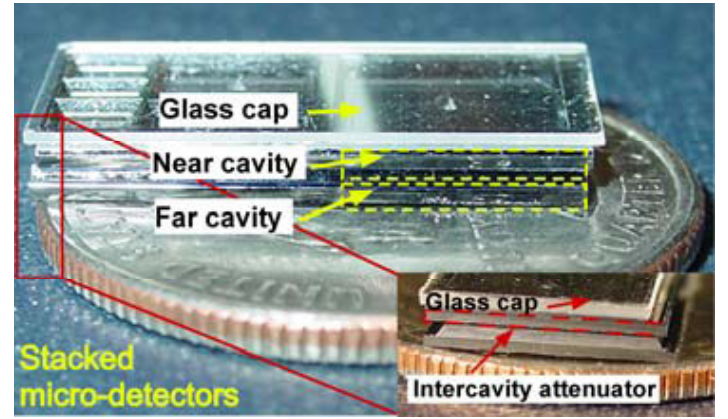

(b)

Figure 5. (a) A composite chip containing multiple micro-detectors with volumes that are orders of magnitude smaller than traditional tubes. (b) Photograph of stacked micro-detectors showing the two-tiered structure and the intercavity attenuator.

future wafer-level gas packaging using $\mathrm{Si} / \mathrm{Au}$ eutectic bonding $[37,38]$. A photograph of a composite die in figure 5(a) shows six separate micro-detector cavities.

The fabrication process for the stacked micro-detector (shown in figure 1(b)) is similar to the basic device process, but requires an additional step for aligning the detection cavities. It involves two $\mathrm{Si}$ wafers ( $\mathrm{Si} 1$ and $\mathrm{Si} 2$ ) and three glass wafers (G1, G2 and G3). First, mask 1 and mask 2 are used to define the boron diffusion and cavity profiles on each $500 \mu \mathrm{m}$ thick $\langle 100\rangle \mathrm{Si}$ wafer (Si1 and Si2). Second, the glass wafers are machined. Mask 3 can be used optionally to selectively thin and pattern the glass cap (G1) and intercavity attenuator (G2). This customization can provide varying absorption thicknesses that target various beta energy ranges (section 3) on the same device. Perforations are machined in the glass cap (G1), which provides electrical access to the cathodes in the near cavity. Patterned openings are also made in G3 (the glass substrate), to provide electrical contact in the far cavity.

Si1 is aligned to G2 and then anodically bonded to it, and $\mathrm{Si} 2$ to G3. Each $\mathrm{Si} /$ glass structure is etched using EDP, and then $\mathrm{Si} 1 / \mathrm{G} 2$ is stacked above $\mathrm{Si} 2 / \mathrm{G} 3$. The machined glass cap (G1) is stacked above the entire structure. Solder plugs or conductive epoxy are used to seal the machined holes in the glass cap and glass substrate (G1 and G3). A die containing multiple stacked micro-detectors (without bonding) is shown in figure $5(b)$. 


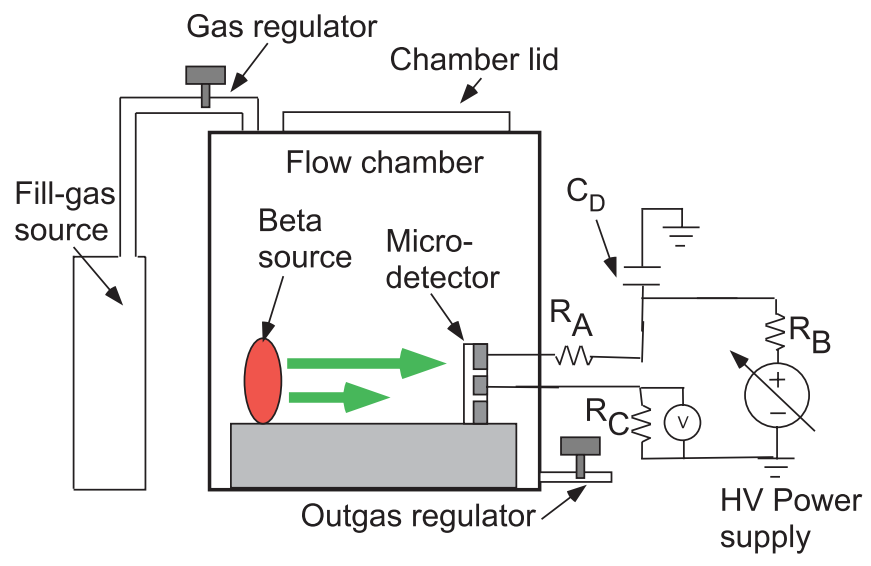

Figure 6. Preliminary test setup for the micro-detector; fill-gas mixture is continually flowed through the chamber during measurements. Inside the chamber, a beta source is positioned at a fixed distance from the device. The bias circuitry includes ballast resistors $\left(R_{A}, R_{B}\right.$, and $\left.R_{C}\right)$ and a discharge capacitor $\left(C_{D}\right)$.

\section{Experimental results}

\subsection{Micro-detector}

The testing setup used to characterize the micro-detectors is shown in figure 6 . The bias circuitry includes ballast resistors located at each electrode and between the anode and the power supply in order to minimize damage from current microdischarges. A discharge capacitor, $C_{D}$, provides the operating bias for the micro-detector. When a beta-particle triggers a microdischarge, $C_{D}$ discharges through the microdetector and lowers the applied bias. The bias drops below the threshold value for avalanche breakdown and the discharge is quenched. By operating near the Geiger-Mueller region, the discharge current pulses can be easily measured without further amplification.

A gas-controlled test chamber was used to evaluate a variety of fill-gases within the sensor, which was operated without the glass cap for the purpose of testing. The fillgases evaluated were $\mathrm{He}, \mathrm{Ne}$, and a mixture of $\mathrm{He}$ and air. Very weak $(0.1-1.0 \mu \mathrm{Ci})$ radioisotope sources $\left({ }^{90} \mathrm{Sr}\right.$ and $\left.{ }^{204} \mathrm{Tl}\right)$ were placed in the chamber to calibrate the detectors. The count rate measured in the He environment showed a slightly higher count rate (10-15\%) compared to the Ne environment. Figure 7 illustrates the relative count rate for various cavity dimensions $\left(8 \times 8 \mathrm{~mm}^{2}, 4 \times 6 \mathrm{~mm}^{2}, 3 \times 6 \mathrm{~mm}^{2}\right.$ and $3 \times$ $3 \mathrm{~mm}^{2}$ ) as a function of source-to-detector distance in a He ambient, while detecting ${ }^{90} \mathrm{Sr}$. As expected, count rates increase with larger detection area and decrease with larger source-to-detector separation.

The micro-detector performance was compared to a commercial general-purpose beta monitor (RPI, Radmonitor: GM1) using a $0.1 \mu \mathrm{Ci}$ source of ${ }^{90} \mathrm{Sr}$ at a distance of $5 \mathrm{~cm}$. As a rule of thumb, the average beta energy can be roughly estimated by [25]

$$
\bar{E}=\frac{1}{3} E_{\beta, \max }(\mathrm{MeV}) .
$$

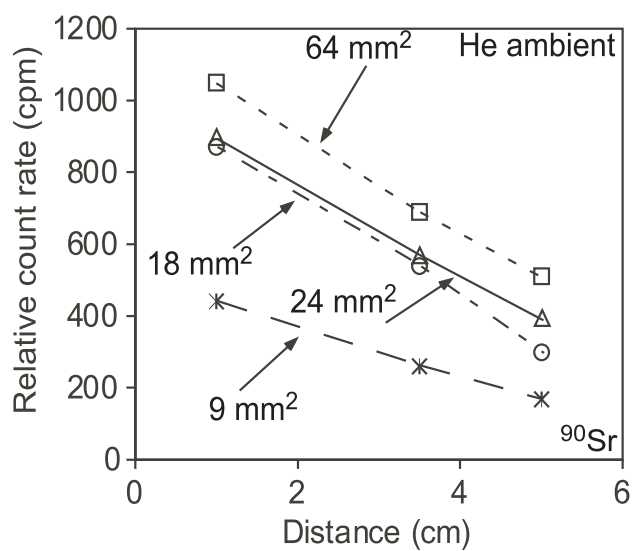

Figure 7. Relative count rates decrease as source-to-detector distances increase. Count rates also decrease with smaller cavity sizes. Four cavity sizes were tested at three separation values using ${ }^{90} \mathrm{Sr}$.

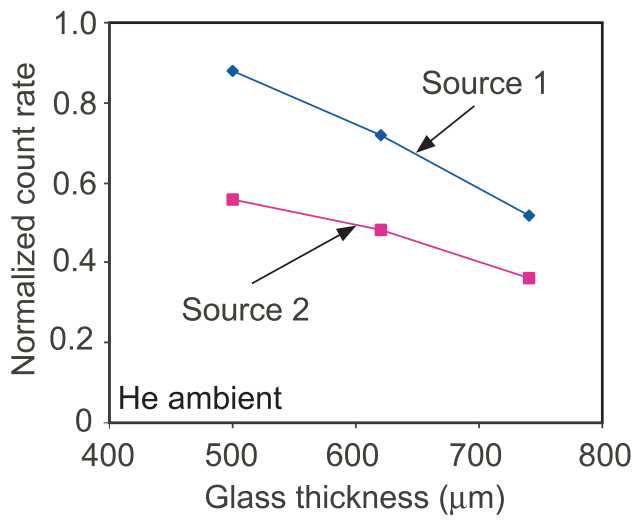

Figure 8. Normalized count rates in the presence of a uranium ore containing two different isotope sources show a decreasing trend (by a factor of about two) as the glass cap thickness increases from $500 \mu \mathrm{m}$ to $750 \mu \mathrm{m}$.

More rigorously calculated values for the average beta energies of ${ }^{90} \mathrm{Sr}$ and ${ }^{90} \mathrm{Y}$ are $196.4 \mathrm{keV}$ and $935.3 \mathrm{keV}$, respectively [28]. The calculated dose rate under these conditions (assuming ${ }^{90} \mathrm{Sr}$ and ${ }^{90} \mathrm{Y}$ are in equilibrium) is $13.5 \mathrm{mRad} \mathrm{h}^{-1}$. The commercial device, which used a Geiger-Mueller gas tube with a radius of $1.6 \mathrm{~cm}$ and a length of $14 \mathrm{~cm}$, provided $9000 \mathrm{cpm}$; whereas the microdetector, with a detection volume of $6.4 \times 10^{-2} \mathrm{~cm}^{3}$, provided $500 \mathrm{cpm}$. When normalized to detector volume, the microdetector performance appears to be quite encouraging. The operating voltages for the micro-detector $(800 \mathrm{~V})$ and the commercial detector $(600 \mathrm{~V})$ are comparable.

The effect of glass cap thickness on the micro-detector count rate was examined (figure 8). Standard glass thicknesses between $500 \mu \mathrm{m}$ and $750 \mu \mathrm{m}$ were tested. As the cap thickness increases, more beta radiation is absorbed before reaching the fill-gas and the measured count rate decreases. These preliminary results demonstrate simple calibrated energy absorption through Pyrex glass. These data have relevance for the stacked micro-detector, in which the intercavity attenuator has similar behavior. 


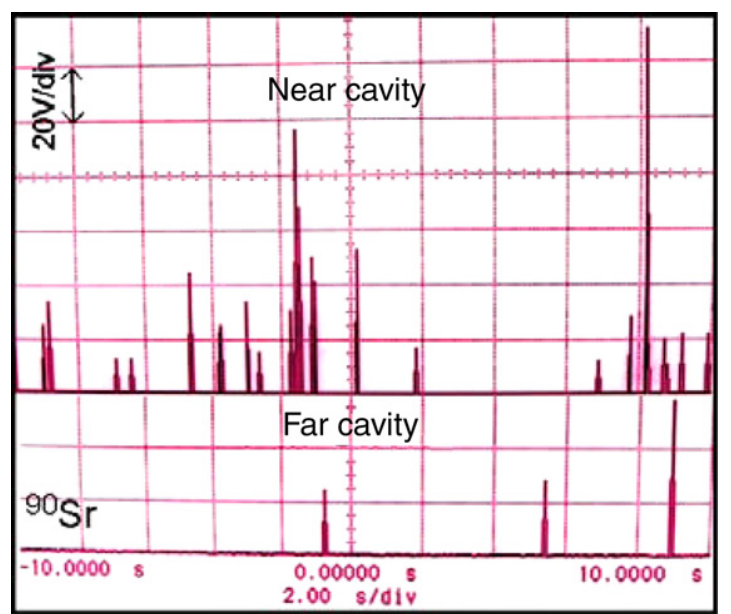

Figure 9. An oscilloscope trace of the upper (near) cavity of the stacked micro-detector shows more pulses per unit time than the lower (far) cavity, for which the glass intercavity attenuator blocks the lower energy particles. Conducted in a Ne ambient.

\subsection{Stacked micro-detector}

Two separate bias circuits (identical to the one used for the micro-detector) and power supplies were attached to each tier of the stacked micro-detector. A $1 \mathrm{~cm}$ spacer was used to maintain the source-to-detector distance. As noted previously, in the stacked micro-detector, higher energy particles are able to penetrate both cavities, while lower energy particles are absorbed in the intercavity attenuator. Preliminary results (figure 9) include an oscilloscope trace of current pulses taken from the near and far cavities with a $500 \mu \mathrm{m}$ thick intercavity attenuator, while in the proximity of $\mathrm{a}^{90} \mathrm{Sr}$ source. The variation in pulse heights within each cavity is due to the statistical variations in breakdown characteristics during operation. It is not associated with operation in the proportional regime because the current pulse amplitudes are much larger than those associated with proportional counters.

Figure 10(a) compares the experimental count ratios for ${ }^{90} \mathrm{Sr}$ and ${ }^{204} \mathrm{Tl}$ with a theoretical estimate using the parameters $E_{C 1}=5 \mathrm{keV}$ and $E_{C 2}=270 \mathrm{keV}$. Radiation with higher endpoint energies is expected to produce higher count rates in both cavities (near and far). ${ }^{204} \mathrm{Tl}$ has a higher endpoint energy $(0.764 \mathrm{MeV})$ compared to ${ }^{90} \mathrm{Sr}(0.546 \mathrm{MeV})$. This corresponds to the higher count ratio measured $(0.42)$ and estimated analytically $(0.31)$ for ${ }^{204} \mathrm{Tl}$ compared to ${ }^{90} \mathrm{Sr}$, which had a measured ratio of 0.14 and an estimated ratio of 0.19 . The error bars indicate the change in count ratios as $E_{C 2}$ is modified. Figure $10(b)$ shows the different isotope signatures for ${ }^{90} \mathrm{Sr}$ and ${ }^{204} \mathrm{Tl}$. The difference in normalized count rates from the near and far cavities can be an initial method of identifying the nature of beta-particles.

\section{Discussion}

In the beta model used to estimate the count ratio, there are several assumptions and simplifications that have been made. For example, it assumes that every beta-particle entering either

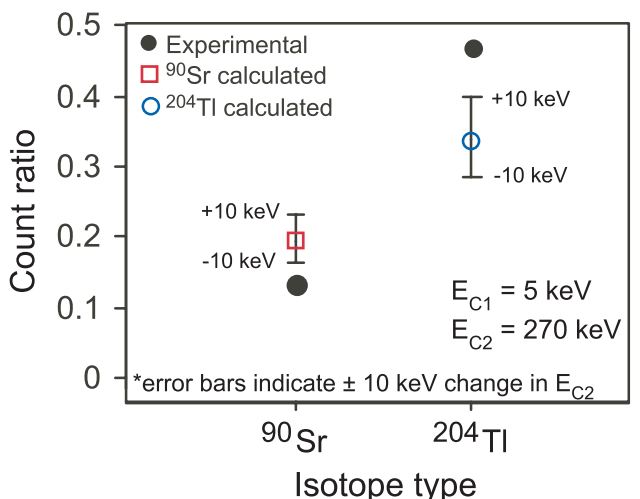

(a)

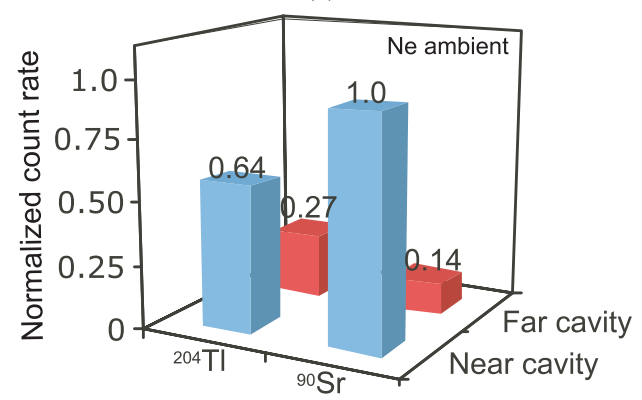

(b)

Figure 10. (a) Using $E_{C 1}=5 \mathrm{keV}$ and $E_{C 2}=270 \mathrm{keV}$, the measured results from the stacked micro-detector were compared with a theoretical estimate. Count ratio is the fraction of measured activity in the far cavity over the near cavity. The intercavity attenuator thickness was $500 \mu \mathrm{m}$. The error bars indicate the change in count ratio with a $10 \mathrm{keV}$ change in $E_{C 2}$. (b) Normalized count rates for the near and far cavities of the stacked micro-detector, while detecting two different isotopes $\left(1.0 \mu \mathrm{Ci}\right.$ of ${ }^{204} \mathrm{Tl}$ and $0.1 \mu \mathrm{Ci}$ of $\left.{ }^{90} \mathrm{Sr}\right)$. The near cavity consistently detected more particles than the far cavity. This suggests that higher energy beta-particles are detected in both cavities, while lower energy particles are detected in the near cavity alone.

cavity with an energy between $E_{C 1}$ and $E_{C 2}$ will deposit enough energy to initiate a microdischarge. However, betaparticles deposit a statistical spread of energies [31]. In addition, the model assumes that the device is ideally biased in a regime where even a single ionization event will instigate breakdown. The model also assumes that the beta-particles have trajectories that are perpendicular to the detector surface and does not take into account the angle of incidence or scattering effects. In actuality, beta-particles can scatter in any direction or have an angle of incidence such that the particles only interact in the near cavity and never reach the far cavity [39]. Each of these factors may affect the experimental count ratios.

In this work, helium and neon were evaluated as possible fill-gases. Although helium provided a slightly higher count rate $(10-15 \%)$, it could be more challenging to package. Another consideration is the use of fill-gases with larger atomic numbers (e.g., $\mathrm{Kr}$ and $\mathrm{Xe}$ ), which may be easier to package and can increase gamma radiation interaction. One possible option for gas packaging involves using commercial high-voltage packages with a glass cap for die-level, hermetic sealing. Another option is to pattern a metal layer on the glass 
cap wafer and use $\mathrm{Au}-\mathrm{Si}$ eutectic bond rings to provide waferlevel packaging. The metal layer can also serve to alleviate possible charging issues on the glass substrate, however, this effect has not been observed.

\section{Conclusions}

This work has shown that micro-detectors for beta radiation can be fabricated using a very simple two-mask process involving stacks of $\mathrm{Si}$ and glass with detection cavities varying in size from $9 \mathrm{~mm}^{2}$ to $64 \mathrm{~mm}^{2}$, and using $\mathrm{He}$ and $\mathrm{Ne}$ fillgases at atmospheric pressure. Various radioisotopes such as ${ }^{90} \mathrm{Sr},{ }^{204} \mathrm{Tl}$ and a uranium ore were used to evaluate the devices. The devices showed sensitivity that was comparable to a commercial gas-based radiation monitor. The design allows for flexibility in terms of sizing and integration with other types of sensors to form composite structures with greater functionality. For example, a two-tiered structure (stacked micro-detector) with aligned detection cavities could enable simple, front-end energy spectroscopy, providing distinguishable profiles for ${ }^{90} \mathrm{Sr}$ and ${ }^{204} \mathrm{Tl}$. This design can be further extended to include multiple stacks with varying attenuator thicknesses, which can increase the energy resolution of the micro-detector.

\section{Acknowledgments}

This work was supported primarily by the Engineering Research Centers Program of the National Science Foundation under award no. EEC-9986866. The facilities used for this research include the Solid State Electronics Laboratory (SSEL) at the University of Michigan. The authors also thank Mr Ramon Torres-Isea and Professor David Wehe for access to the Advanced Physics Lab and Nuclear Engineering test facilities, respectively. YG acknowledges support through the IR/D program while working at the National Science Foundation. The findings do not necessarily reflect the views of the NSF.

\section{References}

[1] Hansen H H 1983 Measurement of the beta-ray spectra of ${ }^{90} \mathrm{Sr}-{ }^{90} \mathrm{Y}$ Int. J. Appl. Radiat. Isot. 34 1241-7

[2] Knoll G F 2000 Radiation Detection and Measurement (New York: Wiley)

[3] Allier C P, Hollander R W, Sarro P M and van Eijk C W E 2000 Scintillation light read-out by thin photodiodes in silicon wells Nucl. Instrum. Methods Phys. Res. A 442 255-8

[4] Allier C P, Hollander R W, van Eijk C W E, Sarro P M, de Boer M, Czirr J B, Chaminade J P and Fouassier C 2001 Thin photodiodes for a neutron scintillator silicon-well detector IEEE Trans. Nucl. Sci. 48 1154-7

[5] Shah K S, Cirignano L, Grazioso R, Klugerman M, Bennet P R, Gupta T K, Moses W W, Weber M J and Derenzo S E $2002 \mathrm{RbGd}_{2} \mathrm{Br}_{7}$ : ce scintillators for gamma-ray and thermal neutron detection IEEE Trans. Nucl. Sci. 49 1655-60

[6] Pellegrin S, Whitney C and Wilson C G 2006 A multichannel nanoparticle scintillation microdevice with integrated waveguides for alpha, beta, gamma, x-ray and neutron detection Proc. IEEE Int. Workshop on Microelectromechanical Systems (MEMS '06) (Istanbul, Turkey, Jan. 2006) pp 682-5

[7] Audet S A, Schooneveld E M, Wouters S E and Kim M H 1990 High purity silicon soft x-ray imaging sensor Sensors Actuators A 22 482-6

[8] Kemmer J A 1987 Silicon detectors for nuclear radiation IEEE Int. Conf. on Solid-State Sensors and Actuators (Transducers '87) vol A 478 pp 252-7

[9] Wada M, Suzuki J and Ozaki Y 1989 Cadmium telluride $\beta$-ray detector Sensors Actuators 19 227-36

[10] Wunstorf R 1997 Radiation hardness of silicon detectors: current status IEEE Trans. Nucl. Sci. 44 806-14

[11] Bertolucci E, Conti M, Mettivier G, Montesi M C and Russo P 2002 BETAview: a digital ß-Imaging system for dynamic studies of biological phenomena Nucl. Instrum. Methods Phys. Res. 478 109-13

[12] Luke P N 1994 Single-polarity charge sensing in ionization detectors using coplanar electrodes Appl. Phys. Lett. 65 2884-6

[13] Lehner C E, He Z and Knoll G F 2003 Intelligent gamma-ray spectroscopy using 3-D position-sensitive detectors IEEE Trans. Nucl. Sci. 50 1090-7

[14] Kiff S D, He Z and Tepper G C 2005 A new coplanar-grid high-pressure xenon gamma-ray spectrometer IEEE Trans. Nucl. Sci. 52 2932-9

[15] Francke T and Vladimir P 2003 Micropattern gaseous detectors 42nd INFN ELOISATRON Project Workshop (Erice, Italy, Sept. 2003)

[16] Oed A 1988 Position-sensitive detector with microstrip anode for electron multiplication with gases Nucl. Instrum. Methods Phys. Res. A 263 351-9

[17] Charpak G, Derre J, Giomataris Y and Rebourgeard P 2002 MICROMEGAS, a multipurpose gaseous detector $\mathrm{Nucl}$. Instrum. Methods Phys. Res. 478 26-36

[18] Sarvestani A, Besch H J, Junk M, MeiBner W, Pavel N, Sauer N, Stiehler R, Walenta A H and Menk R H 1998 Gas amplifying hole structures with resistive position encoding: a new concept for a high rate imaging pixel detector $\mathrm{Nucl}$. Instrum. Methods Phys. Res. A 419 444-51

[19] Sauli F 2001 Gas detectors: achievements and trends Nucl. Instrum. Methods Phys. Res. A 461 47-54

[20] Wilson C G and Gianchandani Y B 2004 MicroGeiger: a microfabricated gas-based beta particle detector Proc. Solid-State Sensors, Actuators and Microsystems Workshop (Hilton Head '04), (Hilton Head Island, SC, June 2004)

[21] Wilson C G, Eun C K and Gianchandani Y B 2005 A microfabricated beta-particle detector with dual cavities for energy spectroscopy Proc. IEEE Int. Workshop on Micro-electromechanical Systems (MEMS '05) (Miami Beach, FL, Jan. 2005) pp 622-5

[22] Wilson C G, Gianchandani Y B, Arslanbekov R R, Kolobov V and Wendt A E 2003 Profiling and modeling of dc nitrogen microplasmas J. Appl. Phys. 94 2845-51

[23] Breskin A 2000 Advances in gas avalanche radiation detectors for biomedical applications Nucl. Instrum. Methods Phys. Res. A 454 26-39

[24] Sauli F 2002 Micro-pattern gas detectors Nucl. Instrum. Methods Phys. Res. A 477 1-7

[25] Martin J 2006 Physics for Radiation Protection (New York: Wiley-VCH)

[26] Turner J E 1995 Atoms, Radiation, and Radiation Protection (New York: Wiley)

[27] Berger M J, Coursey J S, Zucker M A and Chang J 1998 Stopping-power and range tables for electrons, protons, and helium ions http://physics.nist.gov/ PhysRefData/Star/Text/contents.html (ESTAR database)

[28] Firestone R B 2001 http://ie.lbl.gov/toi/radSearch.asp (Isotopes Project) 
[29] Firestone R B 2008 private communication

[30] Skramstad H K and Loughridge D H 1936 The primary ionization of high energy electrons in nitrogen and neon Phys. Rev. 50 677-81

[31] Ibarra M R and Garcia-Torano E 2006 Calculation of electron deposition in proportional counters Appl. Radiat. Isot. 64 1193-7

[32] Brode R B 1939 The specific ionization of high speed particles Rev. Mod. Phys. 11 222-30

[33] Petersen K 1982 Silicon as a mechanical material Proc IEEE 70 420-57

[34] Belloy E, Sayah A and Gijs M A 2000 Powder blasting for three-dimensional microstructuring of glass Sensors Actuators A 86 231-7

[35] Zheng Z-P, Cheng W-H, Huang F-Y and Yan B-H 2007 3D microstructuring of pyrex glass using the electrochemical discharge machining process J. Micromech. Microeng. 17 960-6

[36] West J and Jadhav A 2007 ECDM methods for fluidic interfacing through thin glass substrates and the formation of spherical microcavities J. Micromech. Microeng. 17 403-9

[37] Wolffenbuttel R F 1997 Low-temperature intermediate Au-Si wafer bonding; eutectic or silicide bond Sensors Actuators 62 680-6

[38] Harpster T J and Najafi K 2003 Field-assisted bonding of glass to $\mathrm{Si}-\mathrm{Au}$ eutectic solder for packaging applications Proc. IEEE Int. Workshop on Micro-electromechanical Systems (MEMS '03) (Jan. 2003) pp 630-3

[39] Spalek A 1982 Energy and angular distributions of electrons emitted from spectrometer sources: Monte Carlo calculations Nucl. Instrum. Methods 198 399-402 